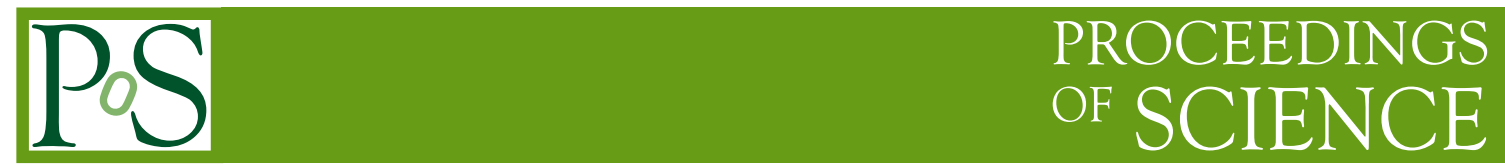

\title{
Determination of the physical parameters of molecular clouds
}

\author{
Riccardo Cesaroni* \\ INAF-Osservatorio Astrofisico di Arcetri, Firenze, Italy \\ E-mail: cesaearcetri.astro.it
}

The most common methods to derive some of the physical parameters of Galactic molecular clouds are described in this lecture. Special attention is devoted to the derivation of the (column) density, mass, and temperature. Advantages and limitations of the different techniques are discussed.

2nd MCCT-SKADS Training School. Radio Astronomy: fundamentals and the new instruments August 26-September 4, 2008

Siguenza, Spain

\footnotetext{
* Speaker.
} 


\section{Introduction}

The goal of this lecture is to describe the most common methods employed to estimate some critical parameters of molecular clouds, from line and continuum observations. In particular, we will discuss the derivation of the velocity field, temperature, (column) density, and mass.

All the information we need is "hidden" in the solution of the radiative transfer equation. For illustrative purposes, it is convenient to consider the solution for the line emission in the simplest case (constant temperature and density along the line of sight and gaussian source structure) and in the Rayleigh-Jeans approximation:

$$
T_{\mathrm{MB}}(v)=\frac{\Omega_{\mathrm{S}}}{\Omega_{\mathrm{S}}+\Omega_{\mathrm{B}}} T_{\mathrm{ex}}\left(1-\mathrm{e}^{-\tau(v)}\right) .
$$

Here, $\Omega_{\mathrm{S}}$ is the solid angle subtended by the source, $\Omega_{\mathrm{B}}$ the solid angle of the instrumental beam, $T_{\mathrm{ex}}$ the gas excitation temperature of the transition, and $\tau$ the line optical depth given by the expression

$$
\tau(v)=\frac{h v}{4 \pi} B_{u l} N_{u}\left(\mathrm{e}^{\frac{h v}{k T_{\mathrm{ex}}}}-1\right) \phi\left(v-v_{0}(V)\right)
$$

with $h$ Planck constant, $k$ Boltzmann constant, $B_{u l}$ Einstein coefficient for stimulated emission, $N_{u}$ column density in the upper level of the transition, $\phi$ normalised line profile, and $v_{0}(V)$ frequency at the line center. Note that the latter is a function of the velocity $V$ along the line of sight of the gas particle emitting the line, according to the Doppler law $v_{0}(V)=v_{\text {rest }}(1-V / c)$, with $v_{\text {rest }}$ line frequency as measured in the laboratory and $c$ speed of light.

Equations (1.1) and (1.2) contain 3 observables $\left(T_{\mathrm{MB}}, \Omega_{\mathrm{S}}\right.$, and $\left.v\right)$ and depend on the physical parameters of the gas. In the following, we will illustrate how measurements of the former make it possible to obtain the latter. Suggested reading on this subject consists of [1, 3, \#,

\section{Velocity field}

The information about the gas velocity is contained in the line profile $\phi$. While several effects contribute to shape lines emitted from molecular clouds, such as thermal and turbulent broadening, the macroscopic velocity field often leaves a clear signature in the line profile. For example, if the instrumental beam encompasses the whole emitting region (i.e. at low angular resolution), the expanding bipolar outflows powered by many newly formed stars are recognizable because they lead to the formation of broad line wings. Instead, the material infalling towards the star can be detected in the form of a so-called "inverse P-Cygni" profile, characterised by a pair of features, of which the red-shifted one is in absorption. This depends partly on the presence of a (sufficiently bright) continuum source against which one sees absorption.

High angular resolution imaging of the line emission allows investigation of the gas distribution at different velocities, thus making it possible to probe the velocity field of the cloud in great detail. This information can be enriched by observations of different molecular species, sampling regions with different densities, temperatures, and molecular abundances.

The most serious limitation of the method is that only one velocity component (that along the line of sight) is obtainable and that we do not know where the particle emitted at that velocity is located along the line of sight. Also, different clumps of gas lying along the same line of sight 
may be moving with the same $V$, so that the map obtained at a given velocity contains "mixed" information of two (or more) distinct regions. Finally, photons emitted at different velocities may overlap each other because of the intrinsic line width (due to various broadening effects), thus complicating the interpretation of the maps.

\section{Kinetic temperature and column density}

In local thermodynamic equilibrium (LTE), the level populations obey the Boltzmann equation and the excitation temperature is a good approximation of the $\mathrm{H}_{2}$ gas kinetic temperature. The latter can thus be obtained in a straightforward manner from observations of an optically thick line (e.g. ${ }^{12} \mathrm{CO}$ ), because $T_{\mathrm{ex}} \simeq T_{\mathrm{K}}$ and Eq. (1.1) for $\tau \gg 1$ gives

$$
T_{\mathrm{K}} \simeq \frac{\Omega_{\mathrm{S}}+\Omega_{\mathrm{B}}}{\Omega_{\mathrm{S}}} T_{\mathrm{MB}}
$$

Here, $\Omega_{\mathrm{B}}$ is known and $T_{\mathrm{MB}}$ and $\Omega_{\mathrm{S}}$ can be measured from a map of the source with suitable angular resolution.

At the same time one can image the same cloud in an optically thin tracer, e.g. ${ }^{13} \mathrm{CO}$. For $\tau \ll 1$, Eq. (1.1) at the line center (i.e. for $v=v_{0}$ ) can be written as

$$
T_{\mathrm{MB}} \simeq \frac{\Omega_{\mathrm{S}}}{\Omega_{\mathrm{S}}+\Omega_{\mathrm{B}}} T_{\mathrm{K}} \tau \propto \frac{\Omega_{\mathrm{S}}}{\Omega_{\mathrm{S}}+\Omega_{\mathrm{B}}} N_{u}
$$

where we made use of Eq. (1.2) in the limit $h v \ll k T_{\mathrm{K}}$. From $N_{u}$ and $T_{\mathrm{K}}$ one can obtain the total column density of the molecule from the Boltzmann equation:

$$
N_{\text {mol }}=\frac{N_{u}}{g_{u}} Q\left(T_{\mathrm{K}}\right)^{\frac{E_{u}}{k^{k}}}
$$

with $g_{u}$ statistical weight of level $u$ and $Q(T)$ partition function, $Q(T)=\sum_{i} g_{i} \exp \left(E_{i} / k T\right)$.

Note that if two lines of an optically thin species are observed, a priori knowledge of $T_{\mathrm{K}}$ is not necessary because both $T_{\mathrm{K}}$ and $N_{\mathrm{mol}}$ can be obtained simultaneously from the Boltzmann equation:

$$
T_{\mathrm{K}}=\frac{h v}{k} \ln \left(\frac{N_{u}^{\prime} g_{u}}{N_{u} g_{u}^{\prime}}\right) .
$$

This result can be extended to the case of three or more optically thin lines. The relevant expression, obtained from the Boltzmann equation, is

$$
\ln \left(\frac{N_{i}}{g_{i}}\right)=\ln \left(\frac{N_{\mathrm{mol}}}{Q\left(T_{\mathrm{K}}\right)}\right)-\frac{E_{i}}{k T_{\mathrm{K}}} .
$$

In practice, one can produce a so-called "rotation diagram" or "Boltzmann plot" by plotting the lefthand side versus $E_{i} / k$ and determining the best linear fit by varying the quantities $\ln \left[N_{\text {mol }} / Q\left(T_{\mathrm{K}}\right)\right]$ and $1 / T_{\mathrm{K}}$. This allows derivation of $T_{\mathrm{K}}$ and $N_{\mathrm{mol}}$, as illustrated in Fig. 1 .

Despite their simplicity, these methods provide us with satisfactory temperature and column density estimates. However, they are not flawless and a few caveats are in order. First of all, one is interested in the $\mathrm{H}_{2}$ column density, $N_{\mathrm{H}_{2}}$, rather than in $N_{\text {mol }}$, and this depends on the molecular abundance, which is quite uncertain for most species. Also, different lines and even more so 


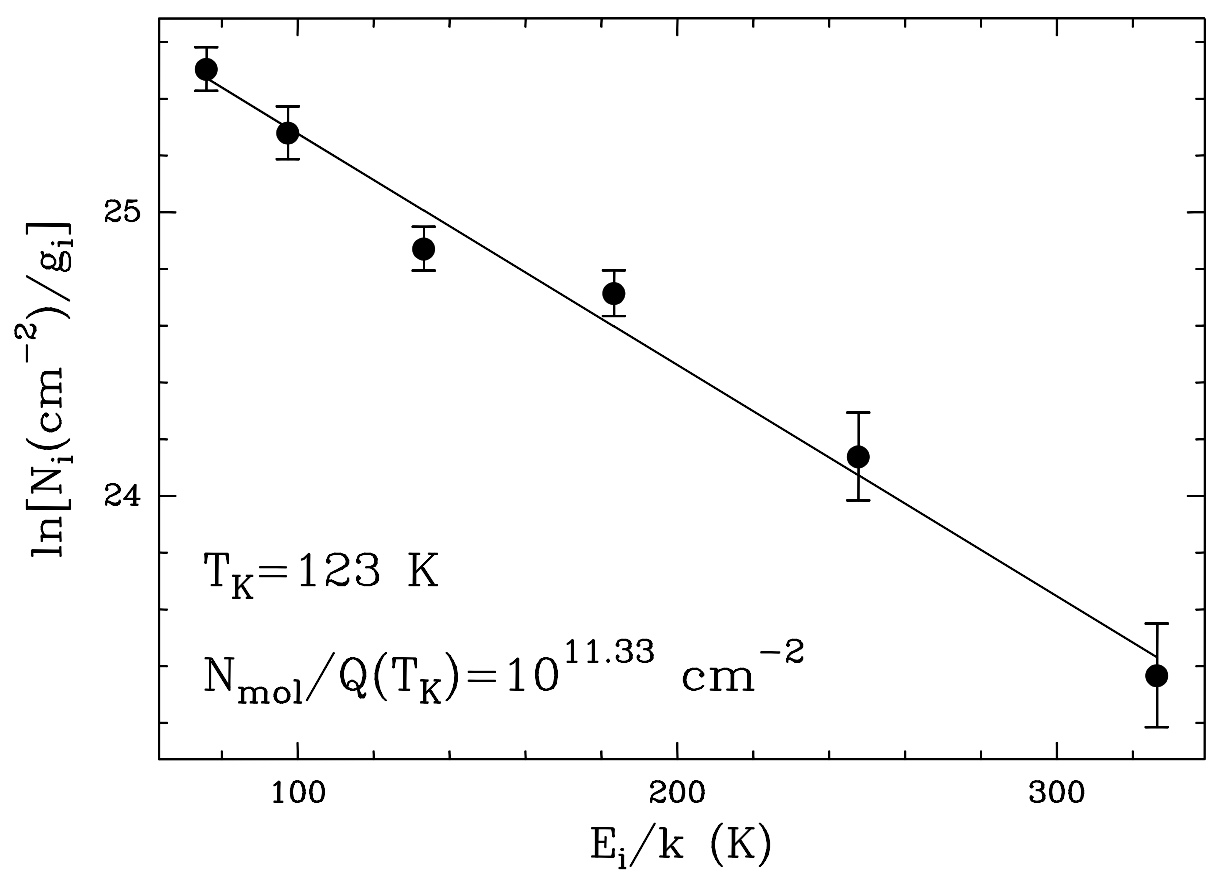

Figure 1: Template rotation diagram and least-square fit (solid line) to the data points. The two best fit parameters are given in the bottom left.

different molecules may trace different environments, with different temperatures and densities. This obviously affects the estimates obtained under the assumption that the gas is homogeneous and isothermal. Moreover, calibration uncertainties introduce errors on $T_{\mathrm{MB}}$ and hence on the derived physical parameters. Estimates obtained from different molecular species can give inconsistent values of $T_{\mathrm{K}}$ and $N_{\mathrm{H}_{2}}$, due to chemical variations inside the cloud. And in all cases one must keep in mind that the estimates obtained are mean values not only along the line of sight, but also inside the instrumental beam solid angle.

\section{Mass and density}

Obtaining a detailed description of the volume density $\left(n_{\mathrm{H}_{2}}\right)$ distribution inside a molecular cloud is a prohibitive task, because all measurable quantities are mean values along the line of sight. An average value of $n_{\mathrm{H}_{2}}$ can be obtained from the ratio between the cloud mass and its volume, under the (naïve) assumption of spherical symmetry. Albeit approximate, this is satisfactory for many purposes and has the advantage of relying upon the estimate of an integrated quantity (the mass), which can be calculated in various ways. In the following, we discuss three methods to derive mass estimates and one method to obtain a direct estimate of the volume density.

\subsection{Mass estimate}

A mass estimate can be obtained by integrating all over the cloud surface the column density obtained as explained in Sect. 3. Clearly, the same caveats as for the determination of $N_{\text {mol }}$ apply here, beside the fact that the mass scales like the cloud surface, namely like the distance squared: 
given the uncertainties on the distance estimates, the mass obtained in this way is likely affected by significant errors.

Another possibility is to assume that the cloud is in hydrostatic equilibrium, when the gravitational forces are balanced by the gas internal pressure. Then the mass must equal the so called “virial” mass (see e.g. [2]), namely $M_{\mathrm{vir}} \propto d \theta \Delta V^{2}$, with $d$ distance, $\theta$ angular radius of the cloud, and $\Delta V$ line width. The dependence on the distance is less important than for the previous estimate, but the assumption of equilibrium is questionable and should rather be verified a posteriori than assumed a priori.

The best estimate is probably that obtainable from the dust continuum emission at (sub)millimeter wavelengths, where the cloud is optically thin. To a good approximation, one has $M \propto S_{v} d^{2} /\left(\kappa T_{\mathrm{K}}\right)$, where $S_{v}$ is the flux density of the cloud and $\kappa$ the dust absorption coefficient. The obvious caveats are the presence of temperature gradients and the uncertainties on the dust properties.

\subsection{Density estimate}

The last method discussed here consists in a direct estimate of $n_{\mathrm{H}_{2}}$. This is possible with measurements of at least two lines of a molecular species that is not in LTE conditions. In fact, as already seen in Sect. B, under LTE conditions the level populations obey the Boltzmann equation and depend only on the gas temperature. On the other hand, if the gas density is sufficiently low (below the "critical" value defined by the ratio between the Einstein coefficient for spontaneous emission and the corresponding collisional coefficient), the molecule may not be in LTE and the line intensities depend also on the density. Therefore, it is conceptually possible to make use of the measured line intensities to derive an estimate of $n_{\mathrm{H}_{2}}$.

In practice, this result can be achieved by comparing the measured line brightness temperatures with those computed by a numerical code calculating the level populations of the given molecule as a function of the physical parameters of the gas - i.e. $T_{\mathrm{K}}, n_{\mathrm{H}_{2}}$, the velocity gradient and the molecular abundance relative to $\mathrm{H}_{2}$. An example of the method is given in Fig. \&. Here, we consider the case of the two rotational transitions $J=2 \rightarrow 1$ and $J=5 \rightarrow 4$ of the $C^{34}$ S molecule. In the top panel, the measured brightness temperature of the (2-1) line is plotted as a function of $T_{\mathrm{K}}$ and $n_{\mathrm{H}_{2}}$. Note how the curves become parallel to the $n_{\mathrm{H}_{2}}$ axis as soon as the transition becomes optically thick (shaded area): this is the consequence of the fact that large opacities bring the corresponding level populations to LTE, where only the value of $T_{\mathrm{K}}$ matters. The two red curves allow for the uncertainty on the measured $T_{\mathrm{B}}$ : all pairs $T_{\mathrm{K}}, n_{\mathrm{H}_{2}}$ whose points lie outside these curves are ruled out. Then we also consider the ratio between the brightness temperatures of the (2-1) and (5-4) lines - see middle panel. This sets an additional constraint on $T_{\mathrm{K}}$ and $n_{\mathrm{H}_{2}}$, which is given by the two blue curves: only points lying inside these two curves correspond (within the observational errors) to the observed ratio $T_{\mathrm{B}}(5-4) / T_{\mathrm{B}}(2-1)$.

The bottom panel of Fig. 目 overlays the two allowed regions, showing that only a limited portion of the $T_{\mathrm{K}}-n_{\mathrm{H}_{2}}$ plane (the yellow region) can satisfy the constraints set by both $T_{\mathrm{B}}(2-1)$ and $T_{\mathrm{B}}(5-4) / T_{\mathrm{B}}(2-1)$. Of the two parameters, $n_{\mathrm{H}_{2}}$ appears to be better constrained, due to the fact that $\mathrm{C}^{34} \mathrm{~S}$ is more sensitive to variations of $n_{\mathrm{H}_{2}}$ than $T_{\mathrm{K}}$. This can be seen in the top panel, where the curve in the optically thin part of the plot are almost parallel to the $T_{\mathrm{K}}$ axis: this means that $T_{\mathrm{B}}(2-1)$ is sensitive only to large changes in $T_{\mathrm{K}}$. 


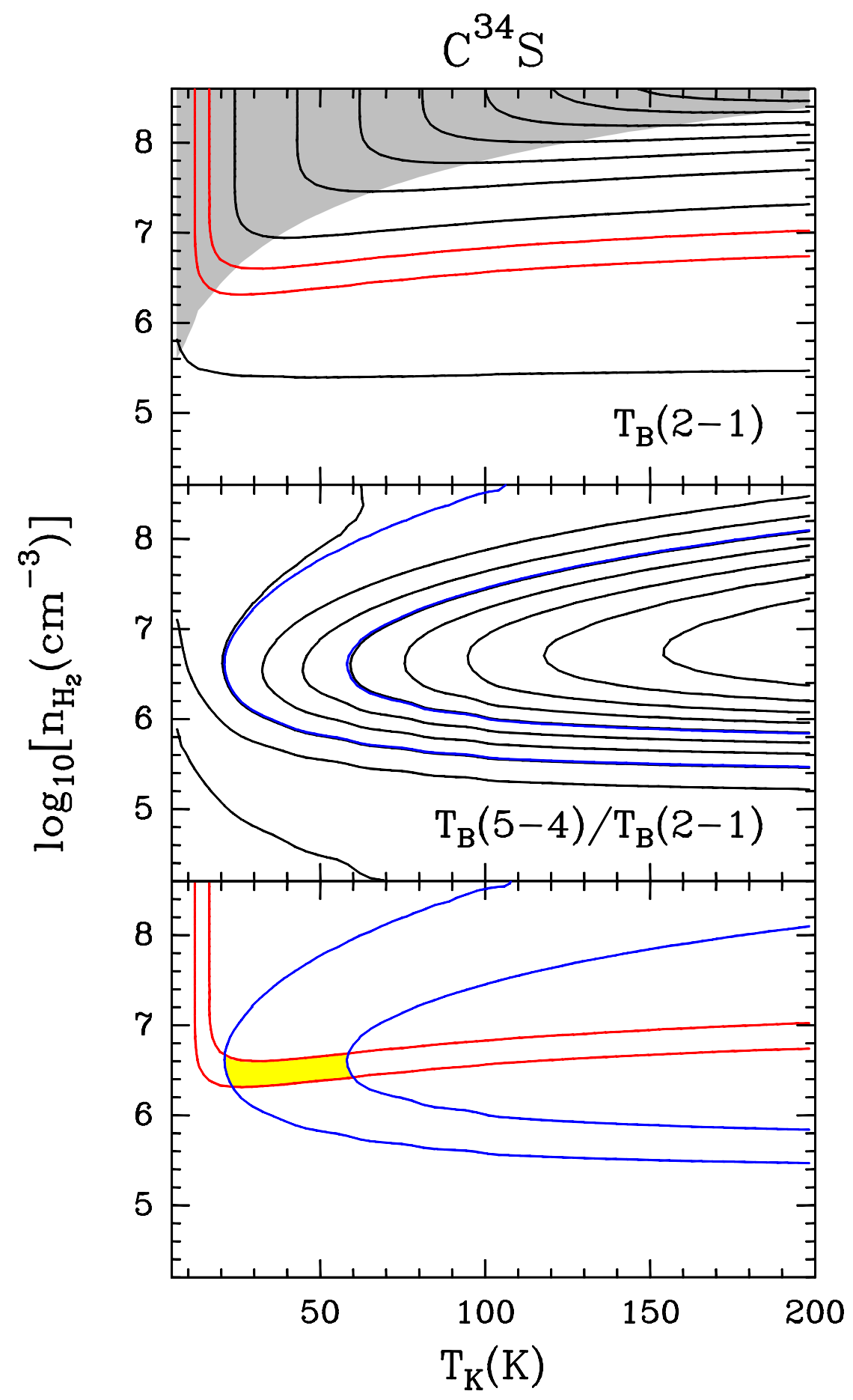

Figure 2: Top: contour plot of the calculated brightness temperature of the $\mathrm{C}^{34} \mathrm{~S}(2-1)$ rotational transition as a function of $\mathrm{H}_{2}$ gas temperature and density. The shaded area indicates the values of $T_{\mathrm{K}}$ and $n_{\mathrm{H}_{2}}$ for which the line opacity is $>1$. The red contours delimit the region corresponding to the measured value of $T_{\mathrm{B}}(2-1)$, allowing for the observational error. Middle: Same as top panel, for the ratio $T_{\mathrm{B}}(5-4) / T_{\mathrm{B}}(2-1)$. The blue curves are the analogous of the red curves in the top panel. Bottom: Overlay of the blue and red contours mentioned above, whose intersection (yellow area) defines the allowed values of $T_{\mathrm{K}}$ and $n_{\mathrm{H}_{2}}$. 
Problems with this method are that the resulting $n_{\mathrm{H}_{2}}$ is model dependent and that in any case it depends also on the assumptions made for other physical parameters, such as the abundance of the molecule and the radiation field. Besides, calibration uncertainties can seriously affect the brightness temperatures and, consequently, the density estimate obtained from them.

One must also consider that the values of $T_{\mathrm{B}}$ depend on the beam filling factor, namely the ratio between the source solid angle and the instrumental beam, which in most cases changes significantly from line to line. In our example, the frequency of the $C^{34} S(5-4)$ line is 2.5 times that of the (2-1), implying a ratio of $\sim 6.2$ between the corresponding beams, if both lines are observed with the same telescope. Unless the the source is extended with respect to both beams, the correction to take into account the beam filling factor is significantly different for the two lines. A reliable determination of these corrections requires a good map of the source.

Last but not least, the method works for a restricted range of densities. As noted above, nonLTE conditions are necessary for this technique and these are attained only if $n_{\mathrm{H}_{2}}$ is less than the critical density. Since the latter depends on the transition, the highest critical density among all the lines observed sets the maximum $n_{\mathrm{H}_{2}}$ that can be estimated. Observations of more transitions and/or different molecular species may be needed to explore larger values of $n_{\mathrm{H}_{2}}$.

\section{References}

[1] R. Genzel, Physical Conditions and Heating/Cooling Processes in High Mass Star Formation Regions, in The Physics of Star Formation and Early Stellar Evolution, NATO ASI 342 (1991) 155

[2] I. MacLaren, K.M. Richardson, A.W. Wolfendale, Corrections to virial estimates of molecular cloud masses, ApJ, 333 (1988) 821

[3] C.M. Walmsley, Physical Conditions in Dense Cores, in Galactic and Extragalactic Star Formation, NATO ASI 232 (1988) 181

[4] T.L. Wilson, C.M. Walmsley, Small-scale clumping in molecular clouds, A\&AR, 1 (1989) 141 